\title{
Structural basis for inhibition of the RNA-dependent RNA polymerase from SARS-CoV-2 by remdesivir
}

\author{
Wanchao Yin ${ }^{1,2 *}$, Chunyou Mao ${ }^{2 *}$, Xiaodong Luan ${ }^{3,4,5 *}$, Dan-Dan Shen ${ }^{2 *}$, Qingya Shen ${ }^{2 *}$, Haixia Su ${ }^{1,6 *}$, \\ Xiaoxi Wang', Fulai Zhou', Wenfeng Zhao', Minqi Gao ${ }^{7}$, Shenghai Chang ${ }^{8,9}$, Yuan-Chao Xie', Guanghui Tian', \\ He-Wei Jiang ${ }^{10}$, Sheng-Ce Tao ${ }^{10}$, Jingshan Shen ${ }^{1,6}$, Yi Jiang ${ }^{1,6}$, Hualiang Jiang ${ }^{1,6}$, Yechun Xu1 ${ }^{1,6}+$, \\ Shuyang Zhang ${ }^{4,5,3}+$, Yan Zhang ${ }^{2,11}+$, H. Eric Xu ${ }^{1,6} \uparrow$
}

\begin{abstract}
${ }^{1}$ The CAS Key Laboratory of Receptor Research, Shanghai Institute of Materia Medica, Chinese Academy of Sciences, Shanghai 201203, China. ${ }^{2}$ Department of Biophysics, and Department of Pathology of Sir Run Run Shaw Hospital, Zhejiang University School of Medicine, Hangzhou 310058, China. ${ }^{3}$ School of Medicine, Tsinghua University, Haidian District, Beijing, China. ${ }^{4}$ Department of Cardiology, Peking Union Medical College Hospital, Peking Union Medical College and Chinese Academy of Medical Sciences, Beijing, China. ${ }^{5}$ Tsinghua-Peking Center for Life Sciences, Tsinghua University, Beijing, China. ${ }^{6}$ University of Chinese Academy of Sciences, Beijing 100049 , China. ${ }^{7}$ WuxiBiortus Biosciences Co. Ltd., 6 Dongsheng West Road, Jiangyin 214437, China. ${ }^{8}$ Center of Cryo-Electron Microscopy, Zhejiang University School of Medicine, Hangzhou 310058, China. ${ }^{9}$ Center of Diagnostic Electron Microscopy, Sir Run Run Shaw Hospital, Zhejiang University School of Medicine, Hangzhou 310058, China.

${ }^{10}$ Shanghai Center for Systems Biomedicine, Key Laboratory of Systems Biomedicine (Ministry of Education), Shanghai Jiao Tong University, Shanghai 200240, China. ${ }^{11}$ Key Laboratory of Immunity and Inflammatory Diseases of Zhejiang Province, Hangzhou 310058, China.
\end{abstract}

*These authors contributed equally to this work.

†Corresponding author. Email: eric.xu@simm.ac.cn (H.E.X.); zhang_yan@zju.edu.cn (Y.Z.); shuyangzhang103@nrdrs.org (S.Z.); ycxu@simm.ac.cn (Y.X.)

The pandemic of Corona Virus Disease 2019 (COVID-19) caused by SARS-CoV-2 has become a global crisis. The replication of SARS-CoV-2 requires the viral RNA-dependent RNA polymerase (RdRp), a target of the antiviral drug, Remdesivir. Here we report the cryo-EM structure of the SARS-CoV-2 RdRp either in the apo form at $2.8 \AA$ resolution or in complex with a 50-base template-primer RNA and Remdesivir at $2.5 \AA$ resolution. The complex structure reveals that the partial double-stranded RNA template is inserted into the central channel of the RdRp where Remdesivir is covalently incorporated into the primer strand at the first replicated base pair and terminates chain elongation. Our structures provide critical insights into the mechanism of viral RNA replication and a rational template for drug design to combat the viral infection.

The COVID-19 pandemic caused by the virus SARS-CoV-2 has become a humanitarian crisis, with over 1.5 million infections and 87,000 death as reported on April 8 of $2020(1,2)$, which has increased rapidly to over 2.99 million infections and 207,000 death on April 27 of 2020 (2). SARS-CoV-2 is closely related to severe acute respiratory syndrome coronavirus (SARS-CoV) and several members from the beta coronavirus family, including bat and pangolin coronaviruses (3-5). Possibly due to stronger binding affinity of the virus spike protein for the host receptor (6-10), SARS-CoV-2 has much higher incidence of human to human transmission, resulting in infection worldwide.

SARS-CoV-2 is a positive strand RNA virus. Its replication is mediated by a multi-subunit replication/transcription complex of viral non-structural proteins (nsp) (11). The core component of this complex is the catalytic subunit (nsp12) of an RNA-dependent RNA polymerase (RdRp) $(12,13)$. By itself, nsp12 has little activity and its functions require accessory factors including nsp7 and nsp8 $(14,15)$, which increase RdRp template binding and processivity. RdRp is also proposed to be the target of a class of antiviral drugs that are nucleotide analogs, including Remdesivir (16-18), which is a prodrug that is converted to the active drug in the triphosphate form
(RTP) within cells (19). As such, RdRp has been a subject of intensive structural biology efforts. The structures of nsp7, nsp8, and the complex of nsp12-nsp7-nsp8 have been determined $(15,20-23)$, providing the overall architecture of the RdRp complex. However, the drug discovery effort is hampered because there is no structure of the SARS-CoV-2 RdRp in complex with an RNA template or with nucleotide inhibitors. Here we determined two cryo-EM structures of the SARS-CoV-2 RdRp complex either in the apo form or in a complex with a template-primer RNA and the antiviral drug Remdesivir.

For cryo-EM studies, we co-expressed nsp12 with nsp7 and nsp8 to form the core RdRp complex in insect cells (Fig. 1A and fig. S1, A to D). The stoichiometric ratio of nsp7 and nsp8 appeared to be less than nsp12, thus additional bacterially expressed nsp7 and nsp8 were supplemented before the final purification step to improve the yield of heterotrimeric complex. The purified nsp12 alone showed little activity in binding to a 50-base partial double-stranded template-primer RNA (Fig. 1B and fig. S1E), which is similar to the SARS-CoV nsp12 (14). The presence of nsp7 and nsp8 dramatically increased nsp12 binding to the template-primer RNA (fig. S1E). The nsp12-nsp7-nsp8 complex also showed RNA 
polymerization activity on a poly-U template upon addition of adenosine triphosphate (ATP) (Fig. 1, B and C). This RNA polymerization activity was effectively inhibited by the addition of the active triphosphate form of Remdesivir (RTP) (Fig. 1D). Even in the presence of $10 \mathrm{mM}$ ATP, $1 \mathrm{mM}$ RTP completely inhibited RdRp polymerization activity. In contrast, Remdesivir, as a prodrug, at $5 \mathrm{mM}$ concentration, did not have any inhibitory effect on the polymerization activity of the purified enzyme (fig. S1F), neither did Remdesivir in its monophosphate form (RMP) (fig. S1G).

The purified RdRp complex is relatively thermostable with a melting temperature of $53^{\circ} \mathrm{C}$ (fig. $\left.\mathrm{S} 1 \mathrm{H}\right)$. Negative-stain EM visualization of the nsp12-nsp7-nsp8 complex displayed monodispersed particles with excellent homogeneity (fig. S1I). For the apo nsp12-nsp7-nsp8 complex, we vitrified the sample in the presence of the detergent DDM. The initial attempt at image processing revealed that the particles are preferentially oriented (fig. S2A). Therefore, we collected over 7,400 micrograph movies of more than 5.7 million particle projections to increase the number of projection from the non-preferential orientation. Of these, 81,494 particles were used to yield a density map of $2.8 \AA$ resolution (fig. S2, B and E). Cryo-EM studies of the nsp12-nsp7-nsp8 complex bound with the template-primer RNA and RTP (termed as the template-RTP RdRp complex) faced two challenges (fig. S3). First, most particles were adsorbed to cryo-EM grid bars rather than staying in the vitreous ice. Second, the RNA duplex were dissociated from the template-RTP RdRp complex likely due to conditions of cryo-EM specimen preparation. Eventually, we prepared the cryo-EM specimen of the template-RTP RdRp complex at $15 \mathrm{mg} / \mathrm{ml}$, much higher than normal concentrations used for EM studies of soluble protein complexes. The high concentration of the complex has a mass action effect to stabilize the RNA-protein complex and has excess amount of the complex to escape the absorption of cryo-EM grid bars to enter the vitreous ice (fig. S3). We collected 2,886 micrograph movies, which yielded a $2.5 \AA$ resolution structure using 130,386 particle projections. Because of the relatively high resolution of our structure, the EM map is clear for key structural features across the complex (fig. S4, A to F).

The structure of the apo RdRp complex contains one nsp12, one nsp7 and two nsp8, with an overall arrangement resembling those seen in SARS-CoV and the recently solved structure of SARS-CoV-2 $(15,23)$ (Fig. 2, A and B). Different from the SARS-CoV RdRp structure but similar to the recent SARS-CoV-2 RdRp structure, our structure reveals that nsp12 also contains an N-terminal $\beta$-hairpin (residues 31-50) and an extended nidovirus RdRp-associated nucleotidyl-transferase domain (NiRAN, residues 115-250) (24), with seven helices and three $\beta$-strands $(15,23)$. Following the NiRAN domain is an interface domain (residues 251-365) composed of three helices and five $\beta$-strands, which is connected to the RdRp domain (residues 366-920) (Figs. 1A and 2B). The nsp12 RdRp domain displays the canonical cupped right-handed configuration (25), with the finger subdomain (resides 397-581 and residues 621-679) forming a closed circle with the thumb subdomain (residues 819-920) (Fig. 2, A and B). The closed conformation is stabilized by the binding of nsp7 and nsp8, with one nsp8 molecule sitting on the top of the finger subdomain and interacting with the interface domain. The closed conformation of nsp12 is further stabilized by the nsp7-nsp8 heterodimer, which is packed against the thumb-finger interface (Fig. 2, A and B). In addition, we were able to assign two zinc ions in the conserved metal binding motifs composed by H295-C301-C306-C310 and C487-H642-C645-C646 (Fig. 2C), which are also observed in the SARS-CoV RdRp structure (15). These zinc ions likely serve as conserved structural components in maintaining the integrity of the RdRp architecture.

The structure of the template-RTP RdRp complex contains one nsp12, one nsp7 and one nsp8 (Fig. 3, A and B). The second nsp8 was largely invisible in the EM map of the template-RTP complex (fig. S4C), therefore it was not included in the final model. In addition, the template-RTP RdRp structure contains 14-base RNA in the template strand, 11-base RNA in the primer strand, and the inhibitor Remdesivir in its monophosphate form (RMP) (Fig. 3, C and D), which is covalently linked to the primer strand, as well as a pyrophosphate and three magnesium ions that may serve as catalytic ions near the active site (Fig. 3D and fig. S4, D to F) (26).

The overall structure of the template-RTP RdRp complex is similar to the apo RdRp structure, with nsp12 in a closed conformation (Figs. 2A and 3A). The double-stranded RNA helix, formed by 11 base-pairs from the template-primer RNA (Figs. 3C and 4, A to E), is held by the finger-palm-thumb subdomains. Extensive protein-RNA interactions are observed between the template-primer RNA and nsp12, with a total of 29 residues from nsp12 directly participating in the binding of the RNA (Fig. 4E). Surprisingly no RNA interactions are mediated by nsp7 or nsp8 although these two proteins are required for RNA binding by RdRp. Most proteinRNA interactions involve the RNA phosphate-ribose backbones, with many interactions directly to 2 ' $-\mathrm{OH}$ groups (Fig. $4 \mathrm{E}$ ), thus providing a basis to distinguish RNA from DNA. There are no contacts from nsp12 to any base pairs of the template-primer RNA, suggesting a sequence independent binding of RNA by RdRp. This is consistent with the fact that no specific sequence is required for the enzymatic activity of RdRp at the elongation step.

At the $3^{\prime}$ end of the primer strand is Remdesivir monophosphate (RMP) (Figs. 3D and 4, D and E, and fig. S4, E and $\mathrm{F})$, which is covalently incorporated into the primer strand at the +1 position (Fig. 4E). Additional nucleotides at the +2 and +3 positions of the template strand interact with residues from the back of finger subdomain (Fig. 4, A and B). Despite 
the presence of excess RTP in complex assembly, only a single RMP is assembled into the primer strand as observed in the structure. Thus, Remdesivir, like many nucleotide analog prodrugs, inhibits the viral RdRp activity through non-obligate RNA chain termination, a mechanism that requires the conversion of the parent drug to the active triphosphate form $(27,28)$.

The RMP position is at the center of the catalytic active site (Fig. 3D). As an adenosine monophosphate analog, RMP forms base-stacking interactions with upstream base from the primer strand and two hydrogen bonds with the uridine base from the template strand (Fig. 3D and fig. S5). In addition, RMP also forms interactions with side chains from K545 and R555. Near the bound RMP are two magnesium ions and a pyrophosphate. Both magnesium ions interact with the phosphate diester backbone and they are part of catalytic active site. The pyrophosphate is at the gate of the nucleotide entry channel to the active site and may block the entry of nucleotide triphosphate to the active side (Fig. 3, C and D).

The catalytic active site of the nsp12 RdRp is constructed by seven conserved motifs from A to G (Figs. $1 \mathrm{~A}$ and $3 \mathrm{E}$ and fig. S6). Motifs ABCD are from the palm subdomain with an SDD sequence (residues 759-761) in motif $\mathrm{C}$ forming the catalytic active center (Fig. 3D). Both D760 and D761 are involved in coordination of the two magnesium ions at the catalytic center. Motifs $\mathrm{F}$ and $\mathrm{G}$ are located within the finger subdomain and they interact with the template strand RNA and direct this strand into the active site (Fig. 3E). Motif F also interacts with the primer strand RNA with the side chains of K545 and R555 contacting the +1 base (Fig. 3D), thus stabilizing the incoming nucleotide in the correct position for catalysis. The orientation of template-primer RNA in the active site is similar to the orientation of template-primer RNA in the poliovirus RdRp elongation complex (29) and the HCV NS5B RdRp inhibitor complex (30) (fig. S7). The residues involved in RNA binding as well as residues comprising the catalytic active site are highly conserved (31, 32), highlighting the conserved mechanism of genome replication by RdRp in these diverse RNA viruses, and suggesting it may be possible to develop broad spectrum antiviral inhibitors such as Remdesivir (18) and Galidesivir (BCX4430) (33).

Structural comparison reveals several interesting differences between the apo and complex structures (Fig. 3, E and F, and fig. S8, A and B). First, nsp7 moves toward the RdRp core by $1.5 \AA$ (as measured by nsp7 residue F49, fig. S8, A and B), leading to a rearrangement of the interface, which results in weaker association of the second nsp8 in the complex. Second, the loop that connects the first and second helix of the thumb subdomain moves outward by $2.8 \AA$ (as measured by nsp12 residue $\mathrm{I} 847$ ) to accommodate the binding of the double stranded RNA helix (Fig. 3F). Third, motif $G$ residues K500 and S501 also move outward by $2.0 \AA$ to accommodate the binding of the template strand RNA. Outside of these changes, the apo nsp12 and the RNA-complex nsp12 are very similar, with an RMSD of $0.52 \AA$ for all $\mathrm{C} \alpha$ atoms across the whole protein. In particular, the structural elements that make up the catalytic active site can be exactly superimposed (Fig. 3E), suggesting that the SARS-CoV-2 RdRp is a relatively stable enzyme that is ready to function as a replicase upon binding of RNA template. Viral RdRp is a highly processive enzyme with a rate of replication up to 100 nucleotides per second (34). No significant conformational changes between the apo and the active enzyme structures are consistent with the high processivity of the viral RNA polymerase, which does not need to consume additional energy for conformational changes in the active site during the replication cycle.

Besides Remdesivir, several nucleotide analog drugs, including Favipiravir, Ribavirin, Galidesivir, and EIDD-2801, efficiently inhibit SARS-CoV-2 replication in cell-based assays $(35,36)$. Like Remdesivir, these nucleotide analogs are proposed to inhibit the viral RdRp through non-obligate RNA chain termination, a mechanism that requires the conversion of the parent compound to the triphosphate active form (33). The structure of the template-RTP RdRp complex provides an excellent model to rationalize how these drugs inhibit the SARS-CoV-2 RdRp activity (fig. S8C). In particular, EIDD2801 has been shown to be $3-10$ times more potent than Remdesivir in blocking SARS-CoV-2 replication (36). The N4 hydroxyl group off the cytidine ring forms an extra hydrogen bond with the side chain of K545 and cytidine base also forms an extra hydrogen bond with the guanine base from the template strand. These two extra hydrogen bonds may explain the apparent higher potency of EIDD-2801 in inhibiting SARS-CoV-2 replication.

The COVID-19 pandemic has inflicted emotional pain and economic burden across the globe. Enzymes that are vital for the viral life cycle are excellent antiviral drug targets as they are different from the host proteins. Among viral enzymes, RdRp is the major target of many existing nucleotide drugs. In this paper, we report the structure of the SARS-CoV-2 RdRp complex in the apo form and in the complex with a template-primer RNA and the active form of Remdesivir. The structures reveal how the template-primer RNA is recognized by the enzyme and how chain elongation is inhibited by Remdesivir. Structure comparison and sequence alignment suggest that the mode of substrate RNA recognition and Remdesivir inhibition of RdRp is highly conserved in diverse RNA viruses, providing a basis for designing broad spectrum antiviral drugs based on nucleotide analogs. Moreover, our structures provide a solid template for modeling and modifying the existing nucleotide drugs, including the highly potent EIDD-2801. Together, these observations provide a rational basis to design even more potent inhibitors to combat the vicious infection of SARS-CoV-2. 


\section{REFERENCES AND NOTES}

1. A. E. Gorbalenya, S. C. Baker, R. S. Baric, R. J. de Groot, C. Drosten, A. A. Gulyaeva, B. L. Haagmans, C. Lauber, A. M. Leontovich, B. W. Neuman, D. Penzar, S. Perlman, L. L. M. Poon, D. Samborskiy, I. A. Sidorov, I. Sola, J. Ziebuhr, Severe acute respiratory syndrome-related coronavirus: The species and its viruses - a statement of the Coronavirus Study Group. bioRxiv 2020.02.07.937862 (2020). https://doi.org/10.1101/2020.02.07.937862.

2. E. Dong, H. Du, L. Gardner, An interactive web-based dashboard to track COVID-19 in real time. Lancet Infect. Dis. 10.1016/S1473-3099(20)30120-1 (2020). doi:10.1016/S1473-3099(20)30120-1 Medline

3. P. Zhou, X.-L. Yang, X.-G. Wang, B. Hu, L. Zhang, W. Zhang, H.-R. Si, Y. Zhu, B. Li, C.L. Huang, H.-D. Chen, J. Chen, Y. Luo, H. Guo, R.-D. Jiang, M.-Q. Liu, Y. Chen, X.-R. Shen, X. Wang, X.-S. Zheng, K. Zhao, Q.-J. Chen, F. Deng, L.-L. Liu, B. Yan, F.-X Zhan, Y.-Y. Wang, G.-F. Xiao, Z.-L. Shi, A pneumonia outbreak associated with a new coronavirus of probable bat origin. Nature 579, 270-273 (2020). doi:10.1038/s41586-020-2012-7 Medline

4. T. T.-Y. Lam, M. H.-H. Shum, H.-C. Zhu, Y.-G. Tong, X.-B. Ni, Y.-S. Liao, W. Wei, W. Y.-M. Cheung, W.-J. Li, L.-F. Li, G. M. Leung, E. C. Holmes, Y.-L. Hu, Y. Guan, Identifying SARS-CoV-2 related coronaviruses in Malayan pangolins. Nature (2020). doi:10.1038/s41586-020-2169-0 Medline

5. T. Zhang, Q. Wu, Z. Zhang, Probable Pangolin Origin of SARS-CoV-2 Associated with the COVID-19 Outbreak. Curr. Biol. 30, 1346-1351.e2 (2020). doi:10.1016/i.cub.2020.03.022 Medline

6. R. Yan, Y. Zhang, Y. Li, L. Xia, Y. Guo, Q. Zhou, Structural basis for the recognition of SARS-CoV-2 by full-length human ACE2. Science 367, 1444-1448 (2020). doi:10.1126/science.abb2762 Medline

7. D. Wrapp, N. Wang, K. S. Corbett, J. A. Goldsmith, C.-L. Hsieh, O. Abiona, B. S. Graham, J. S. McLellan, Cryo-EM structure of the 2019-nCoV spike in the prefusion conformation. Science 367, 1260-1263 (2020). doi:10.1126/science.abb2507 Medline

8. J. Lan, J. Ge, J. Yu, S. Shan, H. Zhou, S. Fan, Q. Zhang, X. Shi, Q. Wang, L. Zhang, X. Wang, Structure of the SARS-CoV-2 spike receptor-binding domain bound to the ACE2 receptor. Nature 10.1038/s41586-020-2180-5 (2020). doi:10.1038/s41586-020-2180-5 Medline

9. J. Shang, G. Ye, K. Shi, Y. Wan, C. Luo, H. Aihara, Q. Geng, A. Auerbach, F. Li, Structural basis of receptor recognition by SARS-CoV-2. Nature 10.1038/s41586020-2179-y (2020). doi:10.1038/s41586-020-2179-y Medline

10. A. C. Walls, Y.-J. Park, M. A. Tortorici, A. Wall, A. T. McGuire, D. Veesler, Structure, Function, and Antigenicity of the SARS-CoV-2 Spike Glycoprotein. Cell 181, 281292.e6 (2020). doi:10.1016/j.cell.2020.02.058 Medline

11. J. Ziebuhr, The coronavirus replicase. Curr. Top. Microbiol. Immunol. 287, 57-94 (2005). doi:10.1007/3-540-26765-4 3 Medline

12. D. G. Ahn, J. K. Choi, D. R. Taylor, J. W. Oh, Biochemical characterization of a recombinant SARS coronavirus nsp12 RNA-dependent RNA polymerase capable of copying viral RNA templates. Arch. Virol. 157, 2095-2104 (2012). doi:10.1007/s00705-012-1404-x Medline

13. A. J. te Velthuis, J. J. Arnold, C. E. Cameron, S. H. van den Worm, E. J. Snijder, The RNA polymerase activity of SARS-coronavirus nsp12 is primer dependent. Nucleic Acids Res. 38, 203-214 (2010). doi:10.1093/nar/gkp904 Medline

14. L. Subissi, C. C. Posthuma, A. Collet, J. C. Zevenhoven-Dobbe, A. E. Gorbalenya, E. Decroly, E. J. Snijder, B. Canard, I. Imbert, One severe acute respiratory syndrome coronavirus protein complex integrates processive RNA polymerase and exonuclease activities. Proc. Natl. Acad. Sci. U.S.A. 111, E3900-E3909 (2014). doi:10.1073/pnas.1323705111 Medline

15. R. N. Kirchdoerfer, A. B. Ward, Structure of the SARS-CoV nsp12 polymerase bound to nsp7 and nsp8 co-factors. Nat. Commun. 10, 2342 (2019). doi:10.1038/s41467-019-10280-3 Medline

16. M. Wang, R. Cao, L. Zhang, X. Yang, J. Liu, M. Xu, Z. Shi, Z. Hu, W. Zhong, G. Xiao, Remdesivir and chloroquine effectively inhibit the recently emerged novel coronavirus (2019-nCoV) in vitro. Cell Res. 30, 269-271 (2020). doi:10.1038/s41422-020-0282-0 Medline

17. M. L. Holshue, C. DeBolt, S. Lindquist, K. H. Lofy, J. Wiesman, H. Bruce, C. Spitters, K. Ericson, S. Wilkerson, A. Tural, G. Diaz, A. Cohn, L. Fox, A. Patel, S. I. Gerber, L. Kim, S. Tong, X. Lu, S. Lindstrom, M. A. Pallansch, W. C. Weldon, H. M. Biggs, T. M. Uyeki, S. K. Pillai; Washington State 2019-nCoV Case Investigation Team, First
Case of 2019 Novel Coronavirus in the United States. N. Engl. J. Med. 382, 929936 (2020). doi:10.1056/NEJMoa2001191 Medline

18. T. K. Warren, R. Jordan, M. K. Lo, A. S. Ray, R. L. Mackman, V. Soloveva, D. Siegel, M. Perron, R. Bannister, H. C. Hui, N. Larson, R. Strickley, J. Wells, K. S. Stuthman, S. A. Van Tongeren, N. L. Garza, G. Donnelly, A. C. Shurtleff, C. J. Retterer, D. Gharaibeh, R. Zamani, T. Kenny, B. P. Eaton, E. Grimes, L. S. Welch, L. Gomba, C. L. Wilhelmsen, D. K. Nichols, J. E. Nuss, E. R. Nagle, J. R. Kugelman, G. Palacios, E. Doerffler, S. Neville, E. Carra, M. O. Clarke, L. Zhang, W. Lew, B. Ross, Q. Wang, K. Chun, L. Wolfe, D. Babusis, Y. Park, K. M. Stray, I. Trancheva, J. Y. Feng, 0. Barauskas, Y. Xu, P. Wong, M. R. Braun, M. Flint, L. K. McMullan, S.-S. Chen, R. Fearns, S. Swaminathan, D. L. Mayers, C. F. Spiropoulou, W. A. Lee, S. T. Nichol, T. Cihlar, S. Bavari, Therapeutic efficacy of the small molecule GS-5734 against Ebola virus in rhesus monkeys. Nature 531, 381-385 (2016). doi:10.1038/nature17180 Medline

19. D. Siegel, H. C. Hui, E. Doerffler, M. O. Clarke, K. Chun, L. Zhang, S. Neville, E. Carra, W. Lew, B. Ross, Q. Wang, L. Wolfe, R. Jordan, V. Soloveva, J. Knox, J. Perry, M. Perron, K. M. Stray, O. Barauskas, J. Y. Feng, Y. Xu, G. Lee, A. L. Rheingold, A. S. Ray, R. Bannister, R. Strickley, S. Swaminathan, W. A. Lee, S. Bavari, T. Cihlar, M. K. Lo, T. K. Warren, R. L. Mackman, Discovery and Synthesis of a Phosphoramidate Prodrug of a Pyrrolo[2,1-f][triazin-4-amino] Adenine CNucleoside (GS-5734) for the Treatment of Ebola and Emerging Viruses. J. Med. Chem. 60, 1648-1661 (2017). doi:10.1021/acs.jmedchem.6b01594 Medline

20. Y. Zhai, F. Sun, X. Li, H. Pang, X. Xu, M. Bartlam, Z. Rao, Insights into SARS-CoV transcription and replication from the structure of the nsp7-nsp8 hexadecamer. Nat. Struct. Mol. Biol. 12, 980-986 (2005). doi:10.1038/nsmb999 Medline

21. W. Peti, M. A. Johnson, T. Herrmann, B. W. Neuman, M. J. Buchmeier, M. Nelson, J. Joseph, R. Page, R. C. Stevens, P. Kuhn, K. Wüthrich, Structural genomics of the severe acute respiratory syndrome coronavirus: Nuclear magnetic resonance structure of the protein nsP7. J. Virol. 79, 12905-12913 (2005) doi:10.1128/JVL.79.20.12905-12913.2005 Medline

22. M. A. Johnson, K. Jaudzems, K. Wüthrich, NMR Structure of the SARS-CoV Nonstructural Protein 7 in Solution at pH 6.5. J. Mol. Biol. 402, 619-628 (2010). doi:10.1016/i.jmb.2010.07.043 Medline

23. Y. Gao, L. Yan, Y. Huang, F. Liu, Y. Zhao, L. Cao, T. Wang, Q. Sun, Z. Ming, L. Zhang, J. Ge, L. Zheng, Y. Zhang, H. Wang, Y. Zhu, C. Zhu, T. Hu, T. Hua, B. Zhang, X. Yang, J. Li, H. Yang, Z. Liu, W. Xu, L. W. Guddat, Q. Wang, Z. Lou, Z. Rao, Structure of the RNA-dependent RNA polymerase from COVID-19 virus. Science 10.1126/science.abb7498 (2020). doi:10.1126/science.abb7498 Medline

24. K. C. Lehmann, A. Gulyaeva, J. C. Zevenhoven-Dobbe, G. M. C. Janssen, M. Ruben, H. S. Overkleeft, P. A. van Veelen, D. V. Samborskiy, A. A. Kravchenko, A. M. Leontovich, I. A. Sidorov, E. J. Snijder, C. C. Posthuma, A. E. Gorbalenya, Discovery of an essential nucleotidylating activity associated with a newly delineated conserved domain in the RNA polymerase-containing protein of all nidoviruses. Nucleic Acids Res. 43, 8416-8434 (2015). doi:10.1093/nar/gkv838 Medline

25. S. M. McDonald, RNA synthetic mechanisms employed by diverse families of RNA viruses. WIREs RNA 4, 351-367 (2013). doi:10.1002/wrna.1164 Medline

26. M. J. van Hemert, S. H. E. van den Worm, K. Knoops, A. M. Mommaas, A. E. Gorbalenya, E. J. Snijder, SARS-coronavirus replication/transcription complexes are membrane-protected and need a host factor for activity in vitro. PLOS Pathog. 4, e1000054 (2008). doi:10.1371/journal.ppat.1000054 Medline

27. E. P. Tchesnokov, J. Y. Feng, D. P. Porter, M. Götte, Mechanism of Inhibition of Ebola Virus RNA-Dependent RNA Polymerase by Remdesivir. Viruses 11, 326 (2019). doi:10.3390/v11040326 Medline

28. C. J. Gordon, E. P. Tchesnokov, E. Woolner, J. K. Perry, J. Y. Feng, D. P. Porter, M. Götte, Remdesivir is a direct-acting antiviral that inhibits RNA-dependent RNA polymerase from severe acute respiratory syndrome coronavirus 2 with high potency. J. Biol. Chem. 10.1074/jbc.RA120.013679 (2020). doi:10.1074/ibc.RA120.013679 Medline

29. P. Gong, O. B. Peersen, Structural basis for active site closure by the poliovirus RNA-dependent RNA polymerase. Proc. Natl. Acad. Sci. U.S.A. 107, 22505-22510 (2010). doi:10.1073/pnas.1007626107 Medline

30. T. C. Appleby, J. K. Perry, E. Murakami, O. Barauskas, J. Feng, A. Cho, D. Fox 3rd, D. R. Wetmore, M. E. McGrath, A. S. Ray, M. J. Sofia, S. Swaminathan, T. E. Edwards, Structural basis for RNA replication by the hepatitis $C$ virus polymerase. Science 347, 771-775 (2015). doi:10.1126/science.1259210 Medline 
31. A. J. te Velthuis, Common and unique features of viral RNA-dependent polymerases. Cell. Mol. Life Sci. 71, 4403-4420 (2014). doi:10.1007/s00018-0141695-z Medline

32. S. Venkataraman, B. V. L. S. Prasad, R. Selvarajan, RNA Dependent RNA Polymerases: Insights from Structure, Function and Evolution. Viruses 10, 76 (2018). doi:10.3390/v10020076 Medline

33. T. K. Warren, J. Wells, R. G. Panchal, K. S. Stuthman, N. L. Garza, S. A. Van Tongeren, L. Dong, C. J. Retterer, B. P. Eaton, G. Pegoraro, S. Honnold, S. Bantia, P. Kotian, X. Chen, B. R. Taubenheim, L. S. Welch, D. M. Minning, Y. S. Babu, W. P. Sheridan, S. Bavari, Protection against filovirus diseases by a novel broadspectrum nucleoside analogue BCX4430. Nature 508, 402-405 (2014). doi:10.1038/nature13027 Medline

34. G. Campagnola, S. McDonald, S. Beaucourt, M. Vignuzzi, O. B. Peersen, Structurefunction relationships underlying the replication fidelity of viral RNA-dependent RNA polymerases. J. Virol. 89, 275-286 (2015). doi:10.1128/JVl.01574-14 Medline

35. C.-C. Lu, M.-Y. Chen, Y.-L. Chang, Potential therapeutic agents against COVID-19: What we know so far. J. Chin. Med. Assoc. 10.1097/JCMA.0000000000000318 (2020). doi:10.1097/JCMA.0000000000000318 Medline

36. T. P. Sheahan, A. C. Sims, S. Zhou, R. L. Graham, A. J. Pruijssers, M. L. Agostini, S. R. Leist, A. Schäfer, K. H. Dinnon 3rd, L. J. Stevens, J. D. Chappell, X. Lu, T. M. Hughes, A. S. George, C. S. Hill, S. A. Montgomery, A. J. Brown, G. R. Bluemling, M. G. Natchus, M. Saindane, A. A. Kolykhalov, G. Painter, J. Harcourt, A. Tamin, N. J. Thornburg, R. Swanstrom, M. R. Denison, R. S. Baric, An orally bioavailable broadspectrum antiviral inhibits SARS-CoV-2 in human airway epithelial cell cultures and multiple coronaviruses in mice. Sci. Transl. Med. 12, eabb5883 (2020). doi:10.1126/scitrans/med.abb5883 Medline

37. S. Q. Zheng, E. Palovcak, J.-P. Armache, K. A. Verba, Y. Cheng, D. A. Agard, MotionCor2: Anisotropic correction of beam-induced motion for improved cryoelectron microscopy. Nat. Methods 14, 331-332 (2017). doi:10.1038/nmeth.4193 Medline

38. K. Zhang, Gctf: Real-time CTF determination and correction. J. Struct. Biol. 193 , 1-12 (2016). doi:10.1016/i.jsb.2015.11.003 Medline

39. S. H. Scheres, RELION: Implementation of a Bayesian approach to cryo-EM structure determination. J. Struct. Biol. 180, 519-530 (2012). doi:10.1016/i.jsb.2012.09.006 Medline

40. A. Punjani, J. L. Rubinstein, D. J. Fleet, M. A. Brubaker, cryoSPARC: Algorithms for rapid unsupervised cryo-EM structure determination. Nat. Methods 14, 290-296 (2017). doi:10.1038/nmeth.4169 Medline

41. J. B. Heymann, Guidelines for using Bsoft for high resolution reconstruction and validation of biomolecular structures from electron micrographs. Protein Sci. 27, 159-171 (2018). doi:10.1002/pro.3293 Medline

42. E. F. Pettersen, T. D. Goddard, C. C. Huang, G. S. Couch, D. M. Greenblatt, E. C. Meng, T. E. Ferrin, UCSF Chimera-A visualization system for exploratory research and analysis. J. Comput. Chem. 25, 1605-1612 (2004). doi:10.1002/icc.20084 Medline

43. P. Emsley, K. Cowtan, Coot: Model-building tools for molecular graphics. Acta Crystallogr. D 60, 2126-2132 (2004). doi:10.1107/S0907444904019158 Medline

44. P. D. Adams, P. V. Afonine, G. Bunkóczi, V. B. Chen, N. Echols, J. J. Headd, L.-W. Hung, S. Jain, G. J. Kapral, R. W. Grosse Kunstleve, A. J. McCoy, N. W. Moriarty, R. D. Oeffner, R. J. Read, D. C. Richardson, J. S. Richardson, T. C. Terwilliger, P. H. Zwart, The Phenix software for automated determination of macromolecular structures. Methods 55, 94-106 (2011). doi:10.1016/i.ymeth.2011.07.005 Medline

45. F. F. Soon, K. M. Suino-Powell, J. Li, E.-L. Yong, H. E. Xu, K. Melcher, Abscisic acid signaling: Thermal stability shift assays as tool to analyze hormone perception and signal transduction. PLOS ONE 7, e47857 (2012). doi:10.1371/journal.pone.0047857 Medline

46. E. Harder, W. Damm, J. Maple, C. Wu, M. Reboul, J. Y. Xiang, L. Wang, D. Lupyan, M. K. Dahlgren, J. L. Knight, J. W. Kaus, D. S. Cerutti, G. Krilov, W. L. Jorgensen, R. Abel, R. A. Friesner, OPLS3: A Force Field Providing Broad Coverage of Drug-like Small Molecules and Proteins. J. Chem. Theory Comput. 12, 281-296 (2016). doi:10.1021/acs.jctc.5b00864 Medline

\section{ACKNOWLEDGMENTS}

The cryo-EM data were collected at the Center of Cryo-Electron Microscopy, Zhejiang University. Funding: This work was partially supported by Shanghai Municipal Science and Technology Major Project 2019SHZDZX02 and XDB08020303 to H.E.X.; Zhejiang University special scientific research fund for COVID-19 prevention and control E33 and the National Science Foundation of China 81922071 to Y.Z.; Science and Technology Commission of Shanghai Municipal 20431900100 and Jack Ma Foundation 2020-CMKYGG-05 to H.J. and J.S.; CAMS Innovation Fund for "13th Five-Year" National Science and Technology Major Project for New Drugs2019ZX09734001-002, CAMS Innovation Fund for Medical Sciences No. 2020-I2M-CoV19-001, and Tsinghua University-Peking University Center for Life Sciences 045-160321001 to S.Z.; National Key Research and Development Program of China Grant 2016 YFA0500600 and National Natural Science Foundation of China 31970130 and 3167083 to S.T.; National Key R\&D Program of China 2016YFA0502301 to Y.X.; National Natural Science Foundation 31770796 and National Science and Technology Major Project2018ZX09711002 to Y.J. We also thank MedChemExpress for making Remdesivir. Author contributions: W.Y. designed the expression constructs, purified the RdRp complex, prepared samples for negative stain and data collection toward the structures, and participated in figure and manuscript preparation. X.L. designed RdRp activity assays and Remdesivir inhibition experiments as well as expression constructs of the RdRp complex; C.M. and D.-D.S. evaluated the specimen by negative-stain EM, screened the cryo-EM conditions, prepared the cryo-EM grids and collected cryo-EM images with the help of S.C.; D.-D.S. and C.M. performed density map calculations; Q.S., H.S. and W.Z. participated in the model building and refined the final models; H.W.Z. and S.T. provided nsp7 and nsp8 genes; X.W., F.Z., and M.G. participated in expression, purification and functional assays of the RdRp; Y.-C.X., G.T., and J.S. made Remdesivir triphosphate form; Y.J. participated in experimental design and manuscript editing; H.J. conceived and coordinated the project; S.Z. conceived the project, initiated collaboration with H.E.X., and supervised X.L.; Y.X. analyzed the structure and modeling and participated in figure preparation; Y.Z. supervised Q.S., C.M., and D.-D.S, analyzed the structures and participated in manuscript writing; H.E.X. conceived and supervised the project, analyzed the structures, and wrote the manuscript with inputs from all authors. Competing interests: The authors declare no competing interests. Data and materials availability: Density maps and structure coordinates have been deposited with immediate release. The accession numbers of Electron Microscopy Database and the Protein Data Bank are EMD30209 and PDB ID 7BV1 for the apo RdRp complex; EMD-30210 and PDB ID 7BV2 for the template RNA and Remdesivir bound RdRp complex. Materials are available upon request. This work is licensed under a Creative Commons Attribution 4.0 International (CC BY 4.0) license, which permits unrestricted use, distribution, and reproduction in any medium, provided the original work is properly cited. To view a copy of this license, visit https://creativecommons.org/licenses/by/4.0/. This license does not apply to figures/photos/artwork or other content included in the article that is credited to a third party; obtain authorization from the rights holder before using such material.

\section{SUPPLEMENTARY MATERIALS}

science.sciencemag.org/cgi/content/full/science.abc1560/DC1

Materials and Methods

Figs. S1 to S8

Table S1

References (37-46)

8 April 2020; accepted 28 April 2020

Published online 1 May 2020

10.1126/science.abc1560 


\section{A}

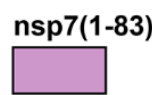

nsp8(1-198)

nsp12(1-932)

$\beta$-hairpin

G $F \quad$ A $\quad$ B $\quad$ C $D$ D

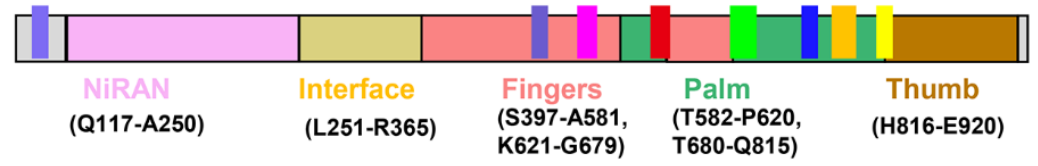

B

\section{SARS-CoV-2}

5. G C U A U G U G A G A U U A A G U U A U 3' direction of primer elongation
| | | | | | | | | | | | | | | | | | | |

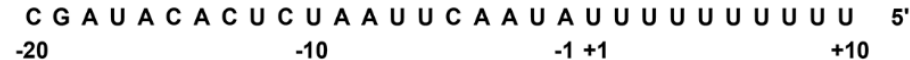

C

D

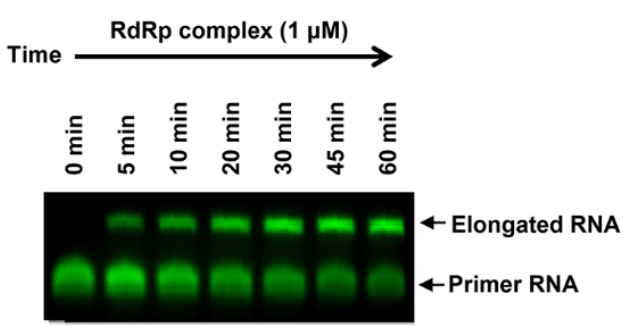

RdRp complex $(1 \mu \mathrm{M})$

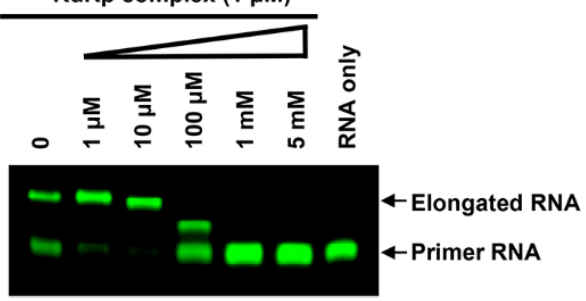

Fig. 1. Assembly of an active nsp12-nsp-7-nsp8 RdRp complex and its inhibition by Remdesivir. (A) Schematic diagram for the components of the RdRp complex, containing nsp12, nsp7 and nsp8. The polymerase motif (A$G$ ) and $\beta$-hairpin unique to the SARS-CoV-2 are highlighted. (B) Sequence of the RNA duplex with a $5^{\prime} U_{10}$ overhang as template for primer extension and RdRp-RNA complex assembly. (C) The recombinant RdRp complex shows polymerase activity in vitro. The primer strand is labeled with fluorescence at the $5^{\prime}$ end. (D) Elongation of partial RNA duplex by the purified RdRp complex and its inhibition by RTP. 
A
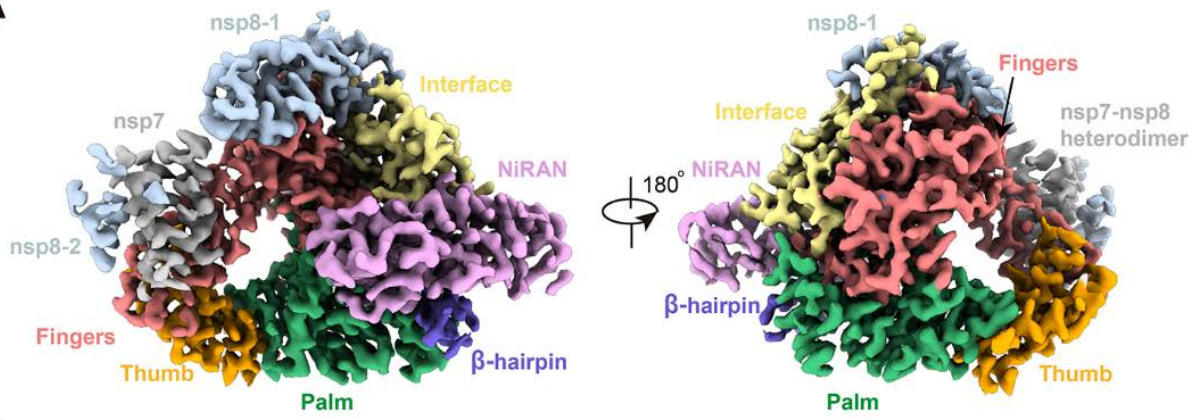

B
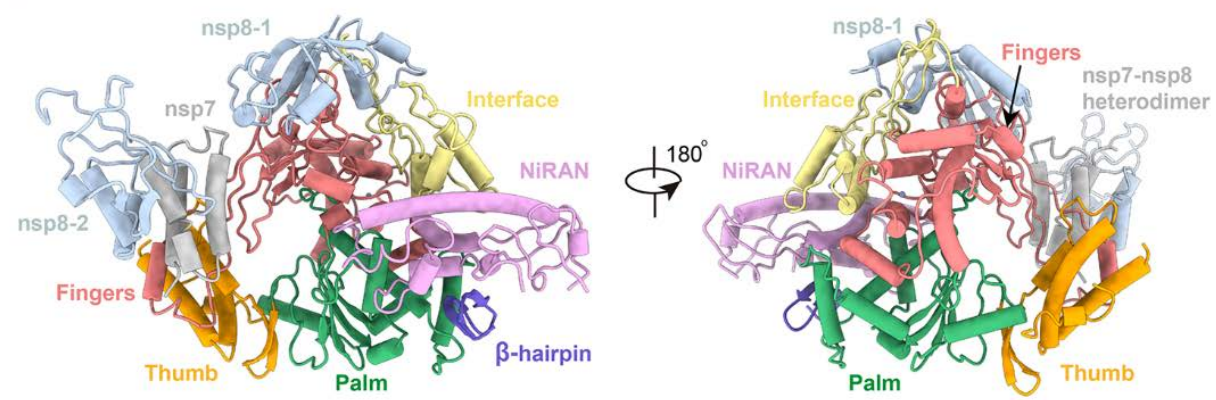

C

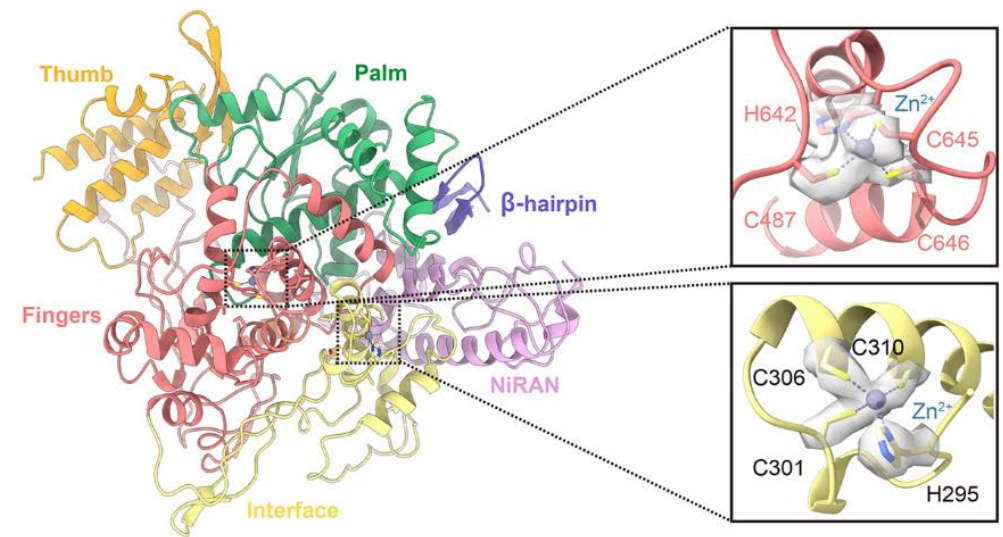

Fig. 2. Cryo-EM Structure of the apo nsp12-nsp-7-nsp8 RdRp complex. ( $A$ and $B$ ) Two views of cryo-EM map (A) and structure (B) of the apo nsp12nsp7-nsp8 complex. The color scheme is according to Fig. $1 \mathrm{~A}$ and is used throughout the paper. (C) The conserved zinc binding motifs are highlighted in the apo structure rendered in ribbon. The coordinate details of the zincbinding residues are shown in stick with the EM map in gray surface. 

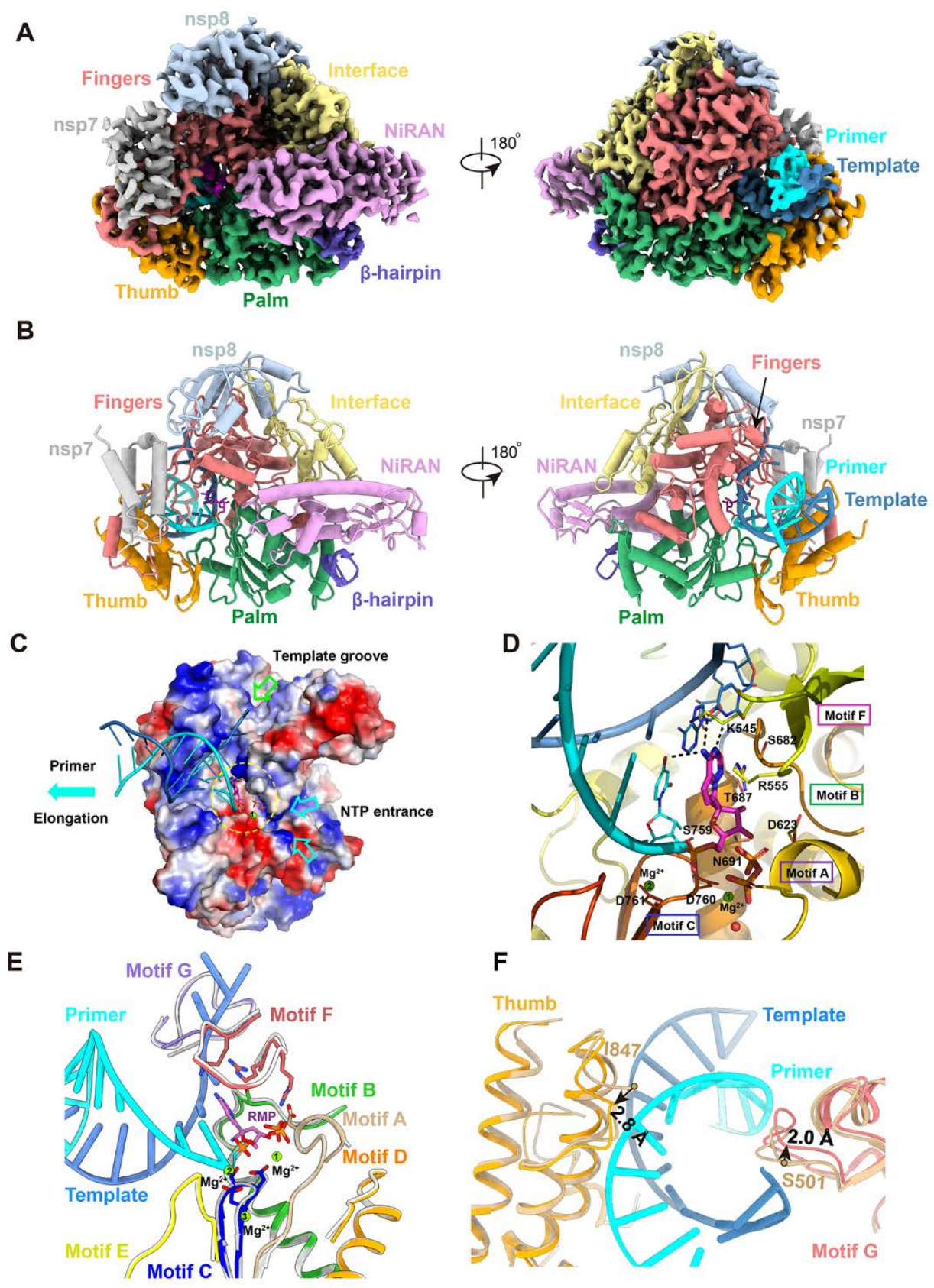

Fig. 3. Cryo-EM Structure of the Remdesivir and RNA bound RdRp complex. (A and $B$ ) Two views of cryo-EM map (A) and structure (B) of the nsp12-nsp7-nsp8 in complex with template-primer RNA and Remdesivir. (C) Surface view of the RdRp active site with the electrostatic potential from red (negative) to blue (positive). For clarity, residues 410-442, 834-919 of nsp12 and nsp8 are excluded from the figure. The covalently bound Remdesivir in the monophosphate form and the product, pyrophosphate, are shown. The active site is emphasized with a yellow dashed circle. The template groove, the entrance for NTP and the elongation direction are annotated with different colored arrows. (D) A close view of the RdRp active site, showing the covalently bound RMP, pyrophosphate, and magnesium ions. Key residues and bases that interact with Remdesivir are shown. (E and $F$ ) Superposition of the conserved RdRp motifs (A to $G$ ) of the RNA bound complex with the apo structure colored in gray, with a close view at the active site (E) and at the exit of the template and primer strand (F). 
A

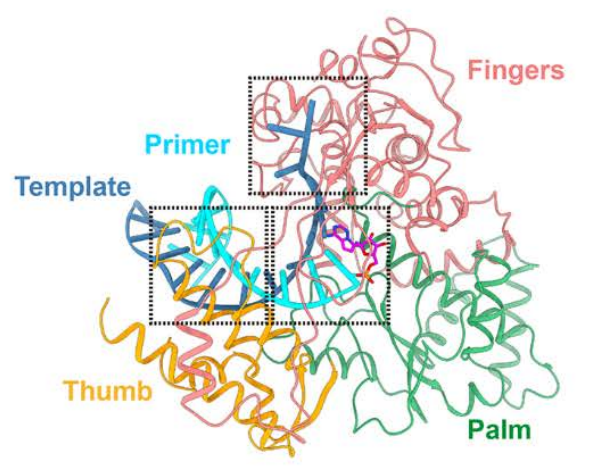

C

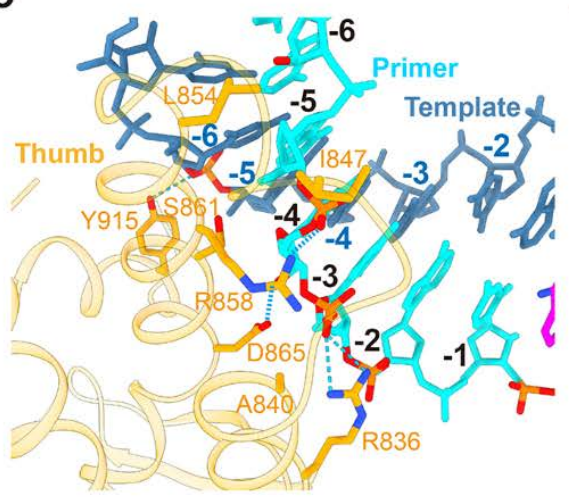

B

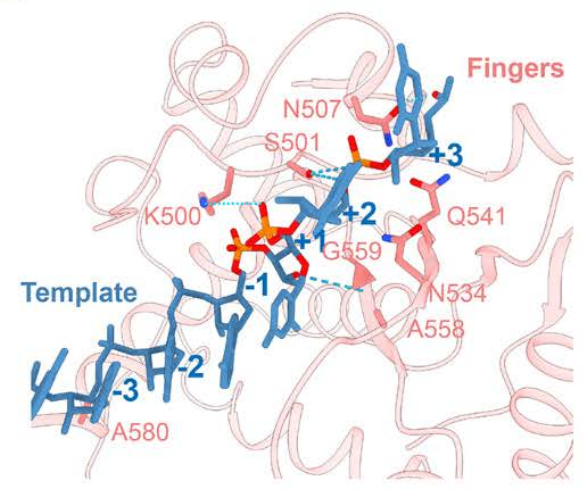

D

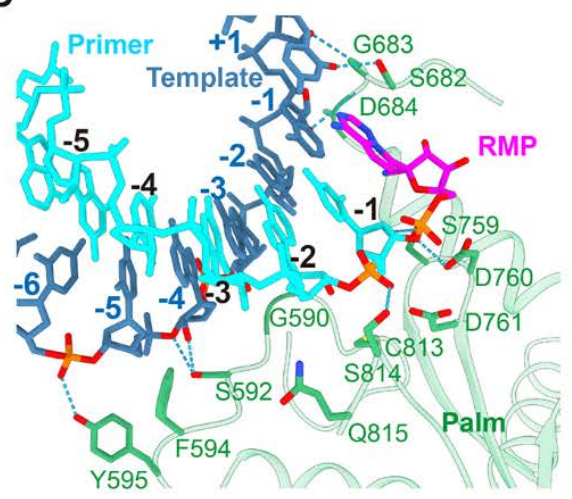

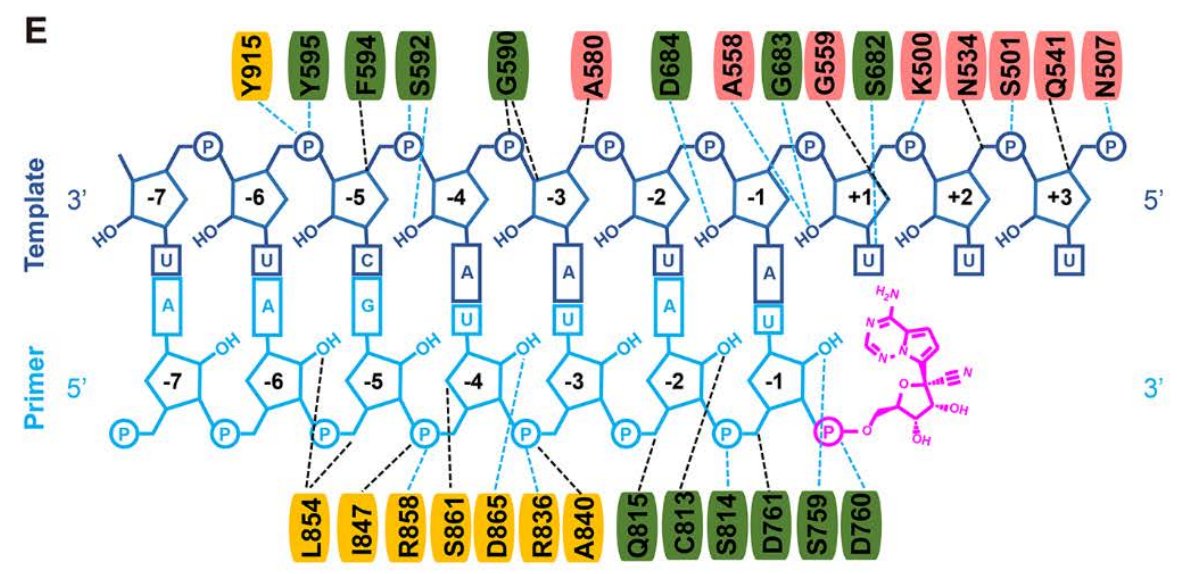

Fig. 4. RNA Recognition by the RdRp complex. (A to D) Protein-RNA interactions in the RNA and Remdesivir bound RdRp complex. (E) Diagram of detailed RNA interactions by RdRp. 